\title{
A Patient Experiencing Bloody Diarrhea and Tenesmus for Three Weeks
}

\author{
Eun Sun $\mathrm{Kim}^{1}$, Yoon Tae Jeen ${ }^{1}$, Joo Young Kim ${ }^{2}$ \\ Departments of Internal Medicine ${ }^{l}$ and Pathology', Korea University College of Medicine, Seoul, Korea
}
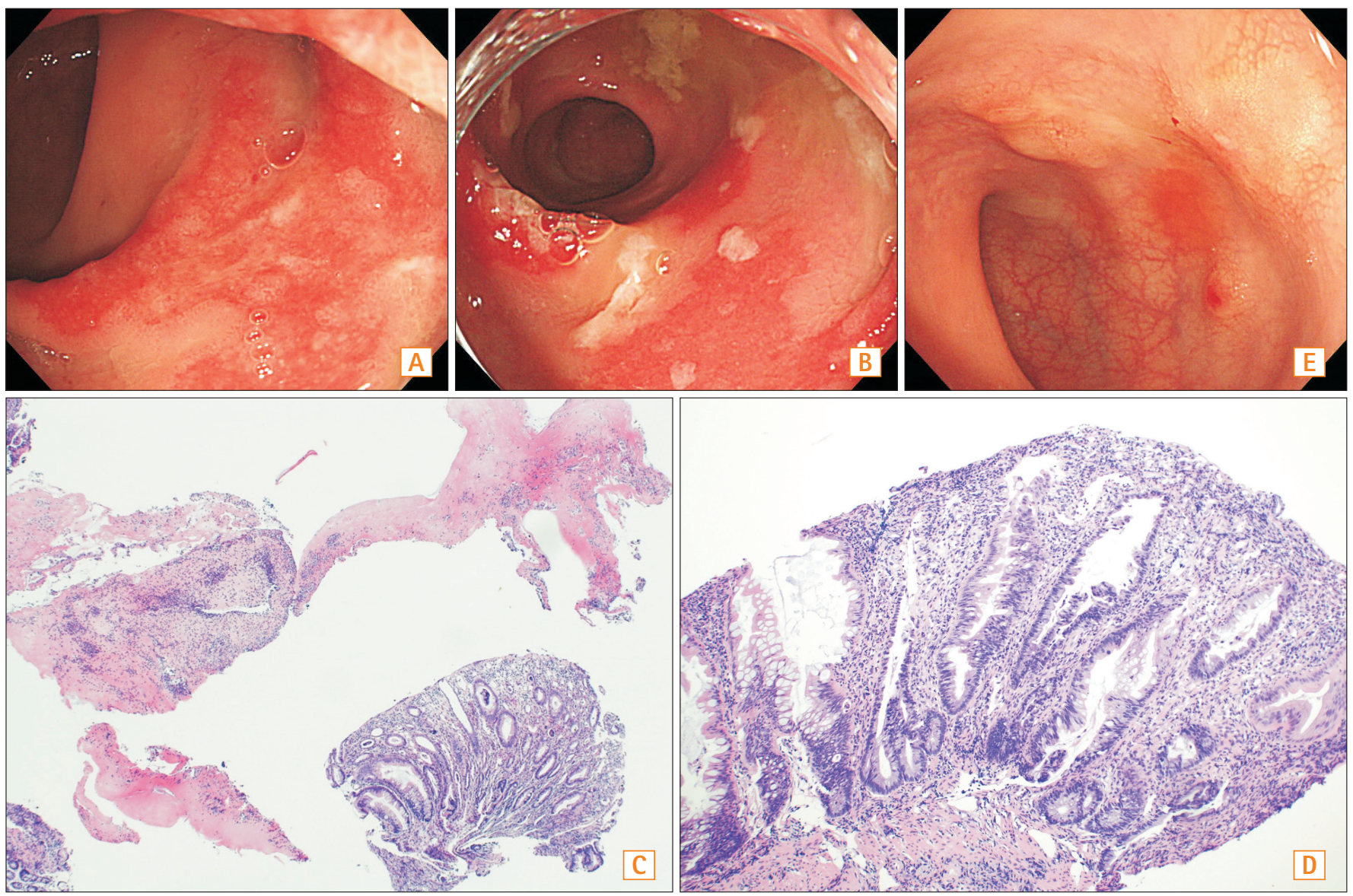

Received March 23, 2015. Accepted March 23, 2015.

Correspondence to Yoon Tae Jeen, Division of Gastroenterology,

Department of Internal Medicine, Korea University Anam Hospital, Korea

University College of Medicine, 73 Inchon-ro, Seongbuk-gu, Seoul 136-705,

Korea. Tel: +82-2-920-6555, Fax: +82-2-923-1943,E-mail: ytjeen@korea.

ac.kr

Financial support: None. Conflict of interest: None.
Question: A 53-year-old woman visited our medical center after experiencing mucous bloody stools, tenesmus, and frequent defecation with lower abdominal pain for the past three weeks. She reported having similar symptoms intermittently for the last two years. On physical examination, the abdomen was mildly distended and soft, with no focal

(c) Copyright 2015. Korean Association for the Study of Intestinal Diseases. All rights reserved.

This is an Open Access article distributed under the terms of the Creative Commons Attribution Non-Commercial License (http://creativecommons.org/licenses/by-nc/3.0) which permits unrestricted non-commercial use, distribution, and reproduction in any medium, provided the original work is properly cited. 
tenderness or palpable mass. Hemoglobin level was $14.6 \mathrm{~g} /$ $\mathrm{dL}$, white blood cell count was $4,190 / \mathrm{mm}^{3}$, platelet count was $228,000 / \mathrm{mm}^{3}$, and inflammatory markers, including erythrocyte sedimentation rate and CRP, were not increased. A stool occult blood test yielded a positive result, but white blood cell and Clostridium difficile toxin assays of stool were negative.

Colonoscopy, performed after complete bowel preparation, showed two polyps in the ascending colon. They were removed using an endoscopic snare and electrocurrent. There were multiple large elevated geographic erythematous lesions with exudate in the rectosigmoid area. The surrounding mucosa was preserved (Fig. A). Multiple endoscopic biopsy specimens were taken from the centers and margins of the lesions in the rectosigmoid area. The pathology report revealed chronic active colitis with crypt abscess and goblet cell depletion. We administered oral metronidazole and ciprofloxacin to control the patient's symptoms, but the patient's symptoms persisted. Follow-up sigmoidoscopy, performed two weeks later, revealed multiple sessile erythematous lesions covered by inflammatory exudates covering in a caplike fashion (Fig. B). The pathology report noted inflammatory exudates and disrupted colonic mucosa (Fig. C). The rectal mucosa showed acute and chronic inflammation in the lamina propria, with a cap of granulation tissue and fibrin covering the surface (Fig. D) The non-neoplastic lesion consisted of elongated hyperplastic crypts (Fig. D). What is the diagnosis for this lesion?

\section{Answer to the Images}

Inflammatory cap polyposis is a rare but distinct disease with characteristic endoscopic and histological findings. ${ }^{1,2}$ Endoscopy shows multiple inflammatory erythematous colonic polyps, consisting of polyposis, located from the rectum to the sigmoid colon. These polyposis-like lesions are covered with fibrinopurulent exudates that appear like a cap. ${ }^{3}$ The most common symptoms are frequent defecation and bloody, mucous diarrhea with abdominal pain and tenesmus. Symptoms last for weeks or months. Women are more frequently afflicted than men. The origin and epidemiology of inflammatory cap polyposis are unknown. Several pathogeneses, including a form of IBD, an infectious origin, and association with mucosal prolapse syndrome or abnormal colonic motility, have been suggested. ${ }^{1,3}$ Diagnosis of this disease is made on the basis of endoscopic and histological findings and clinical manifestations. A specific treatment strategy has not been established. Several case reports have suggested some treatment modalities: anti-inflammatory agents, antibiotics, immunomodulators, and endoscopic and surgical therapy. ${ }^{2,4}$ We tried to resolve the inflammation with a two-week course of mesalazine, but the patient's symptoms worsened. Next, we administered oral beclomethasone for two weeks. The symptoms improved after five days of this treatment. Endoscopy performed one month later showed that the condition had resolved (Fig. E).

\section{REFERENCES}

1. Campbell AP, Cobb CA, Chapman RW, et al. Cap polyposis-an unusual cause of diarrhoea. Gut 1993;34:562-564.

2. Kim ES, Jeen YT, Keum B, et al. Remission of cap polyposis maintained for more than three years after infliximab treatment. Gut Liver 2009;3:325-328.

3. Esaki M, Matsumoto T, Kobayashi H, et al. Cap polyposis of the colon and rectum: an analysis of endoscopic findings. Endoscopy 2001;33:262-266.

4. Bookman ID, Redston MS, Greenberg GR. Successful treatment of cap polyposis with infliximab. Gastroenterology 2004;126:1868-1871. 